\title{
MHD Flow Over a Stretching Permeable Surface Using Homotopy Analysis Method
}

\author{
C. Guled and B. Singh \\ Department of Mathematics, Dr. Babasaheb Ambedkar Technological University, Lonere \\ \{chandrakant.guled@gmail.com; bbsingh@dbatu.ac.in \}
}

\begin{abstract}
The objective of the present paper is to deal with the solution of the third order non-linear boundary layer equation governing the magneto hydrodynamic (MHD) flow of a viscous fluid over a stretching permeable surface using the homotopy analysis method (HAM). The solution obtained has been constructed in the form of series, the convergence of which has been examined carefully by way of plotting convergence control curves. The effect of suction/injection and magnetic parameters on the skin-friction parameter, velocity profiles and stream function have been studied. The results obtained in the present analysis have been compared with the corresponding available results of other researchers. It is observed that the numerical values of the skin-friction for different values of mass transfer and magnetic parameters are convergent and agree well with the earlier existing numerical values. The accuracy in the results shows that the HAM is a very proficient and easily applicable technique for solving differential equations with strong non-linearity.
\end{abstract}

Keywords: MHD flow, homotopy analysis method (HAM), boundary layer flow, suction/injection parameter.

\section{Introduction}

In many areas of applied engineering and industry, we often come across with the boundary layer flow involving a viscid and incompressible fluid over a stretching sheet. This kind of flow process is of interest in the fields of chemical and metallurgical engineering. That is why, it has been widely studied by Anderson et al. [1]. However, from industrial point of view, the magneto-hydrodynamic (MHD) flow problems have recently become more significant. Specifically, many metallurgical processes include the cooling of continuous filaments or strips by extracting them through a motionless fluid. In such metallurgical processes, the fluid properties of the final product would certainly depend on the rate of stretching and the liquid used for cooling. During the process of extracting, these strips sometimes get stretched. It is worth to mention the example of extracting, thinning and strengthening of copper wires.

The characteristics of the final product, however, depend on the rate of cooling to a great extent. A care has to be taken while extracting such strips, in order to control the rate of cooling, in an electrically conducting fluid corresponding to the magnetic field, thereby achieving the expected outcome with desired characteristics. There are many other important applications of hydromagnetics such as extraction of molten metals from the nonmetallic inclusions by using magnetic field, manufacturing of sheeting material through an extrusion process such as hot rolling, glass-fiber manufacturing, plastic sheets through aerodynamic extrusion.

On account of the afore-mentioned industrial applications, it was Pavlov [2] who made the first attempt to investigate the MHD boundary layer flow of an electrically conducting fluid over a stretching wall. Subsequently, the authors [3-11] have also made their contributions to the proposed problem. Nevertheless, so far only a limited attention has been paid by researchers to study the effects of mass transfer parameters e.g. suction and injection on the MHD flow over a stretching porous surface. However, the contributions made by authors $[3,4,7]$ are worthwhile mentioning in this regard. Their studies, however, are confined only to relatively low values of the suction and injection parameters. Thus, the extension of the problem to include large values of the suction and injection parameters remained almost unaddressed. This requirement formed the subject matter of the work of Pop and $\mathrm{Na}$ [12] who made a serious attempt to study the problem of MHD flow of an electrically conducting incompressible fluid over a stretched permeable wall using perturbation technique.

In this study, Pop and $\mathrm{Na}$ identified two extreme cases, namely, large suction and large injection. But, Pop and $\mathrm{Na}$ failed to obtain the solution of the problem for relatively smaller values of the suction and injection parame-

B. Iyer, S. Nalbalwar and R. Pawade (Eds.)

ICCASP/ICMMD-2016. Advances in Intelligent Systems Research.

Vol. 137, Pp. 577-585.

(C) 2017- The authors. Published by Atlantis Press

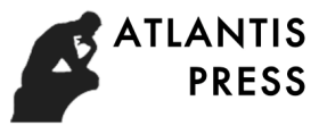

This is an open access article under the CC BY-NC license (http://creativecommons.org/licens)es/by-nc/4) 
ters. Prior to [12], Chakrabarti and Gupta [3] although succeeded in finding the exact solution of the problem as regards the velocity profiles using the similarity solution method, the approach adopted by them was not generic. Instead, the approach to find the solution for velocity profiles was solely based on a guess, subject to the similarity boundary condition, that the velocity of the flow at infinity is zero. So, the approach of Chakrabarti and Gupta is not proficient enough to deal with the solutions of similarity boundary layer equations with strong nonlinearity.

The objective of the present paper, therefore, begins with the necessity of finding an analytical solution of the problem in general by using homotopy analysis method (HAM), so that the effects of suction and injection parameters with any value, large or small, may be investigated in respect of the obtained solution, especially the velocity profiles.

In recent years, analytical solutions of several non-linear problems have been found by researchers [13-37] using HAM which depends on the concept of homotopy in topology (refer [25]). Liao [26], in the year 1992, made an elaborate investigation of the HAM. The strength of HAM lies in the fact that it converges to the analytic series solution of strongly non-linear problems faster than any other existing methods. Further, the rate of convergence and the accuracy of the solution obtained by HAM is not dependent on large/small value of physical parameter(s) involved in the problem, see the reference [27] for details. Moreover, HAM along with the high performance computers and symbolic software leads to a highly accurate analytic solution. The features of the flow field have been analysed and the results obtained in this paper have also been compared with the available results of [3] and [12]. Our results have been found in precise agreement.

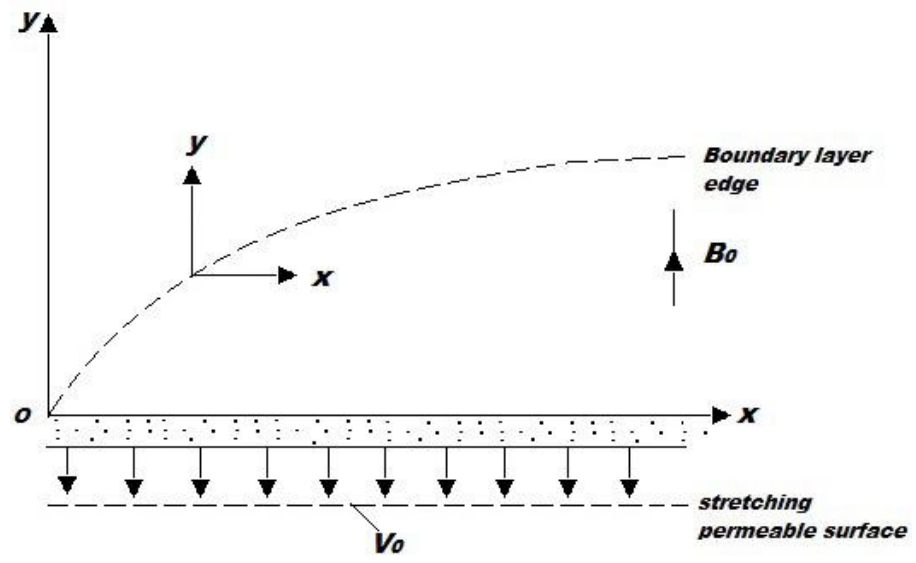

Fig. 1: Physical model of the problem

\section{Governing Equations}

Here the two-dimensional flow of an electrically conducting incompressible fluid under the influence of a uniform magnetic field $B_{0}$ imposed along $y$-axis has been considered. The Fig. 1 illustrates the physical model of the present problem. The flow of the fluid having electrical conductivity $\sigma$ over permeable wall coincides with the plane $y=0$ and the flow is restricted to $y>0$. Keeping the origin fixed, the wall is stretched by introducing two equal and opposite forces along the $x$-axis. The governing equations for the flow problem are therefore (cf. Pop and $\mathrm{Na}[12])$

$$
\begin{aligned}
& \frac{\partial u}{\partial x}+\frac{\partial v}{\partial y}=0 \\
& u \frac{\partial u}{\partial x}+v \frac{\partial u}{\partial y}=v \frac{\partial^{2} u}{\partial y^{2}}-\sigma \frac{B_{0}^{2}}{\rho} u
\end{aligned}
$$

where $u$ and $v$ are the velocity components in the directions of $x$-and $y$-axes, respectively; $v$ is the kinematic viscosity of the fluid, and $\rho$ is the density of fluid. The boundary conditions are

$$
\begin{gathered}
u=a x, \quad v=-v_{0} \text { at } y=0 ; \\
u \rightarrow 0 \text { at } y \rightarrow \infty
\end{gathered}
$$

where $v_{0}$ is either velocity of injection $\left(v_{0}<0\right)$ or velocity of suction $\left(v_{0}>0\right)$, and $a>0$. Here the induced magnetic field has been neglected, which is justifiable for a flow at small magnetic Reynolds number (see the reference [28], p.45). It is also assumed that the external electric field is zero and the electric field due to polarization of charges is negligible. 
On introducing the similarity transformations

$$
\begin{gathered}
u=a x f^{\prime}(\eta), \quad v=-(a v)^{\frac{1}{2}} f(\eta), \\
\eta=\left(\frac{a}{v}\right)^{\frac{1}{2}} y
\end{gathered}
$$

into Eqs. (1)-(3), we obtain the differential equation

together with the boundary conditions

$$
f^{\prime \prime \prime}+f f^{\prime \prime}-f^{\prime 2}-M f^{\prime}=0
$$

$$
f(0)=K, f^{\prime}(0)=1, f^{\prime}(\infty)=0
$$

Here, the primes describe the differentiation with respect to $\eta$,

$K=\frac{V_{0}}{(a v)^{\frac{1}{2}}}$ is either injection $(K<0)$ or suction $(K>0)$ parameter, and

$M=\frac{\sigma B_{0}^{2}}{a \rho}$ is the magnetic parameter.

\section{Homotopy Analysis}

To obtain analytic solution of Eq. (5) subject to the boundary conditions (6), we select $\mathcal{L}$ as

$$
\mathcal{L}=\frac{\partial^{3}}{\partial \eta^{3}}-\gamma \frac{\partial^{2}}{\partial \eta^{2}}
$$

By choosing $q$ as an embedding parameter, we construct the zeroth-order deformation equation as

$$
\begin{gathered}
(1-q) \mathcal{L}\left[f(\eta, \hbar, \gamma ; q)-f_{0}(\eta)\right]=q \hbar H(\eta) \mathcal{N}[f(\eta, \hbar, \gamma ; q)], \\
\eta \in[0,+\infty), \hbar \neq 0, \gamma>0, q \in[0,1],
\end{gathered}
$$

subject to the boundary conditions

$$
\begin{gathered}
f(0, \hbar, \gamma ; q)=K, f^{\prime}(0, \hbar, \gamma ; q)=1, f^{\prime}(+\infty, \hbar, \gamma ; q)=1, \\
\hbar \neq 0, \gamma>0, q \in[0,1]
\end{gathered}
$$

where the primes denote the partial derivatives w.r.t. $\eta$ and

$$
\begin{aligned}
\mathcal{N}[f(\eta, \hbar, \gamma ; q)] & =\frac{\partial^{3} f(\eta, \hbar, \gamma ; q)}{\partial \eta^{3}}+f(\eta, \hbar, \gamma ; q) \frac{\partial^{2} f(\eta, \hbar, \gamma ; q)}{\partial \eta^{2}}-\left(\frac{\partial f(\eta, \hbar, \gamma ; q)}{\partial \eta}\right)^{2} \\
& -M \frac{\partial f(\eta, \hbar, \gamma ; q)}{\partial \eta}
\end{aligned}
$$

When $q=0$, we have from Eq. (8)

$$
\begin{aligned}
& \mathcal{L}\left[f(\eta, \hbar, \gamma ; 0)-f_{0}(\eta)\right]=0 \Rightarrow f(\eta, \hbar, \gamma ; 0)=f_{0}(\eta), \quad \eta \in \\
& {[0,+\infty), \hbar \neq 0, \gamma>0,}
\end{aligned}
$$

and when $q=1$, we have from Eq. (8)

$$
[0,+\infty), \hbar \neq 0, \gamma>0
$$

$$
0=\mathcal{N}[f(\eta, \hbar, \gamma ; 1)] \Rightarrow f(\eta, \hbar, \gamma ; 1)=f(\eta), \eta \in
$$

Hence, $f(\eta, \hbar, \gamma ; q)$ varies from initial solution $f_{0}(\eta)$ to the exact solution $f(\eta)$ as $q$ varies from 0 to 1 .

Keeping in mind the rule of solution expression, we here choose $f_{0}(\eta)$, the initial guess, such that it satisfies $\mathcal{L}\left(f_{0}(\eta)\right)=0$ and the boundary conditions (6). We select

$$
\begin{gathered}
\mathcal{L}\left[C_{1}+C_{2} e^{\gamma \eta}+C_{3} e^{-\gamma \eta}\right]=0 \\
f_{0}(\eta)=\frac{1-e^{-\gamma \eta}}{\gamma}+K, \quad \gamma>0
\end{gathered}
$$

and $H(\eta)=1$. We consider here that the $k$ th-order deformation derivative

$$
f_{0}^{[k]}(\eta, \hbar, \gamma)=\left.\frac{\partial^{k} f(\eta, \hbar, \gamma ; q)}{\partial \eta^{k}}\right|_{q=0},(k \geq 1)
$$

exists. By using Taylor's formula and Eq. (11), we have

$$
f(\eta, \hbar, \gamma ; q)=f_{0}(\eta)+\sum_{k=1}^{+\infty}\left[\frac{f_{0}^{[k]}(\eta, \hbar, \gamma)}{k !}\right] q^{k} .
$$

We assume that both $\hbar$ and $\gamma$ are properly chosen in such a way that the series (16) is convergent at $q=1$. From Eqs. (12) and (14) at $q=1$, we find the following relationship between known initial solution $f_{0}(\eta)$ and the unknown solution $f(\eta)$ : 
where we define

$$
f(\eta)=f_{0}(\eta)+\sum_{k=1}^{+\infty} \frac{f_{0}^{[k]}(\eta, \hbar, \gamma)}{k !}=\sum_{k=0}^{+\infty} \phi_{k}(\eta, \hbar, \gamma)
$$

$$
\phi_{0}(\eta, \hbar, \gamma)=f_{0}(\eta), \quad \phi_{k}(\eta, \hbar, \gamma)=\frac{f_{0}^{[k]}(\eta, \hbar, \gamma)}{k !}, k \geq 1
$$

In order to find the $m$ th-order deformation equation, we first differentiate Eqs. (8) and (9) $m$ times w.r.t. $q$ and then we set $q=0$. Finally, we divide it by $m$ ! to obtain

$$
\mathcal{L}\left[\phi_{m}(\eta)-\chi_{m} \phi_{m-1}(\eta)\right]=\mathcal{N}_{m}(\eta), m \geq 1, \eta \in[0,+\infty),
$$

with the corresponding boundary conditions

and

$$
\phi_{m}(0, \hbar, \gamma)=\phi_{m}^{\prime}(0, \hbar, \gamma), \phi_{m}^{\prime}(+\infty, \hbar, \gamma)=0, m \geq 1, \hbar \neq 0, \gamma>0
$$

$$
\begin{gathered}
\mathcal{N}_{1}(\eta)=\hbar\left[\phi_{0}^{\prime \prime \prime}(\eta, \hbar, \gamma)+\phi_{0}(\eta, \hbar, \gamma) \phi_{0}^{\prime \prime}(\eta, \hbar, \gamma)-\phi_{0}^{\prime 2}(\eta, \hbar, \gamma)-M \phi_{0}^{\prime}(\eta, \hbar, \gamma)\right] \\
\mathcal{N}_{m}(\eta)=\hbar\left[\phi_{m-1}^{\prime \prime \prime}(\eta, \hbar, \gamma)+\sum_{k=0}^{m-1} \phi_{m-1-k}(\eta, \hbar, \gamma) \phi_{k}^{\prime \prime}(\eta, \hbar, \gamma)\right. \\
\left.\quad-\sum_{k=0}^{m-1} \phi_{k}^{\prime}(\eta, \hbar, \gamma) \phi_{m-1-k}^{\prime}(\eta, \hbar, \gamma)-M \phi_{m-1}^{\prime}(\eta, \hbar, \gamma)\right], m>1
\end{gathered}
$$

where primes denote the partial derivative w.r.t. $\eta$.

Using Eqs. (14) and (21), we can first calculate $\mathcal{N}_{1}(\eta)$ and then by solving linear equation (19) with boundary conditions $(20)$, we can find $\phi_{1}(\eta, \hbar, \gamma)$. In the similar manner, we can calculate $\mathcal{N}_{2}(\eta)$ by using Eq. (22) and then find $\phi_{2}(\eta, \hbar, \gamma)$, and so on.

The first order of approximation of Eqs. (5) and (6), calculated by using MATHEMATICA, is:

$$
\begin{aligned}
\phi_{1}(\eta, \hbar, \gamma)=\frac{\hbar}{2 \gamma^{2}} & +\frac{\hbar K}{2 \gamma}+\frac{\hbar M}{2 \gamma^{2}}-\frac{\hbar}{2} \\
& +e^{-\gamma \eta}\left(-\frac{\hbar}{2 \gamma^{2}}+\frac{\gamma \eta \hbar}{2}-\frac{\eta \hbar}{2 \gamma}-\frac{\hbar K}{2 \gamma}-\frac{\eta \hbar K}{2}-\frac{\hbar M}{2 \gamma^{2}}-\frac{\eta \hbar M}{2 \gamma}+\frac{\hbar}{2}\right) .
\end{aligned}
$$

We obtain an analytical solution explicitly for the proposed problem governed by Eqs. (5) and (6) as

$$
f(\eta)=\lim _{N \rightarrow+\infty} \sum_{k=0}^{N} \phi_{m}(\eta, \hbar, \gamma) \text {. }
$$

The solution (24) in the form of infinite series contains two parameters $\hbar(\hbar \neq 0)$ and $\gamma(\gamma>0)$

\section{Convergence of the Analytical Solution}

As pointed out by Liao [29], HAM offers flexibility and freedom to choose better values of $\hbar$ and $\gamma$ which in turn guarantee the convergence of obtained solution in the form of an infinite series to $f(\eta)$. In order to choose $\hbar$, Liao [29] introduced the $\hbar$-curve which gives the admissible range of the parameter $\hbar$ known as convergence region. Further, there exists a best value of the parameter $\gamma$, for every $\hbar$ belonging to the convergence region, for which corresponding series converges fastest.

The second-order derivative of Eq. (24) w.r.t. $\eta$ at $\eta=0$, say,

$$
\sum_{k=0}^{+\infty} \phi_{k}^{\prime \prime}(0, \hbar, \gamma)
$$

must converge if Eq. (24) converges. By using (25), we can find the value of the skin friction coefficient for any value of magnetic parameter $M$ and mass transfer parameter $K$.

The $\hbar$-curves (after 10th order approximation) for the magnetic parameter $M=1$ and for different suction parameters are shown in Fig. 2. From the figure, it is clear that the admissible range for the convergence-control parameter $\hbar$ is $-1 \leq \hbar<0$. 


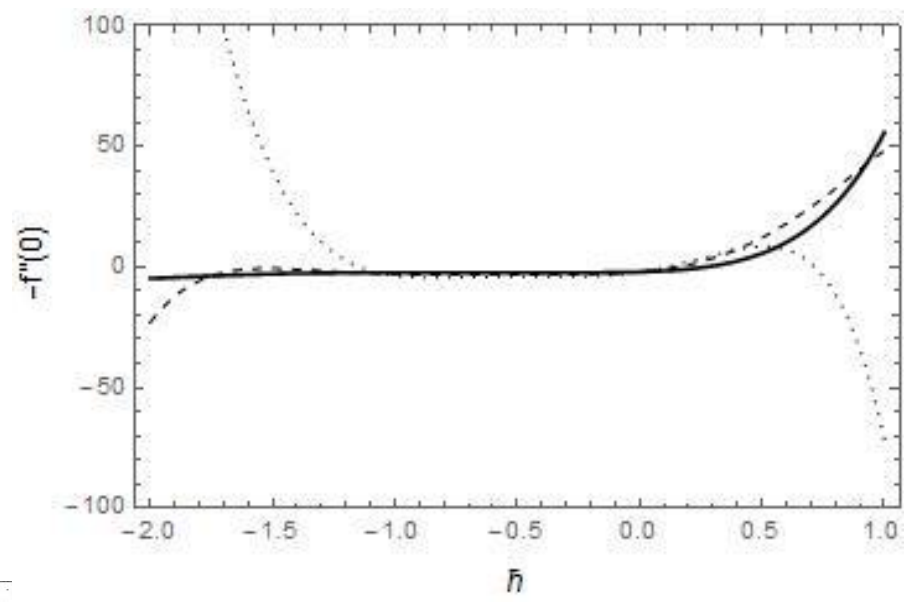

Fig. 2: The $\hbar$-curves for $f$ for $M=1$ and $K=1.5$ (black line), $K=2.5$ (dashed line), $K=3.5$ (dotted line)

\section{Results and discussion}

Table 1: Comparison of the values of $-f^{\prime \prime}(0)$ for different values of $M$ and for suction

\begin{tabular}{|c|c|c|c|c|c|c|c|c|c|c|}
\hline \multirow{3}{*}{$K$} & \multicolumn{5}{|c|}{$M=1$} & \multicolumn{5}{|c|}{$M=2$} \\
\hline & \multicolumn{3}{|c|}{ Present } & \multirow{2}{*}{$\begin{array}{c}\text { Chakrabarti } \\
\text { and Gupta } \\
\text { [3] }\end{array}$} & \multirow[b]{2}{*}{$\begin{array}{l}\text { Pop and } \\
\mathrm{Na}[12]\end{array}$} & \multicolumn{3}{|c|}{ Present } & \multirow{2}{*}{$\begin{array}{c}\text { Chakrabarti } \\
\text { and Gupta } \\
\text { [3] }\end{array}$} & \multirow[b]{2}{*}{$\begin{array}{l}\text { Pop and } \\
\mathrm{Na} \text { [12] }\end{array}$} \\
\hline & $\begin{array}{c}10^{\text {th }} \text { or- } \\
\text { der }\end{array}$ & $\begin{array}{c}15^{\text {th }} \text { or- } \\
\text { der }\end{array}$ & $\begin{array}{c}20^{\text {th }} \text { or- } \\
\text { der }\end{array}$ & & & $\begin{array}{c}10^{\text {th }} \text { or- } \\
\text { der }\end{array}$ & $\begin{array}{c}15^{\text {th }} \text { or- } \\
\text { der }\end{array}$ & $\begin{array}{c}20^{\text {th }} \text { or- } \\
\text { der }\end{array}$ & & \\
\hline 0 & 1.41404 & 1.41421 & 1.41421 & 1.41421 & - & 1.73196 & 1.73205 & 1.73205 & 1.73205 & - \\
\hline 0.5 & 1.68603 & 1.68614 & 1.68614 & 1.68614 & - & 1.98965 & 1.99999 & 2 & 2 & - \\
\hline 1 & 1.98925 & 1.99999 & 2 & 2 & - & 2.30272 & 2.30278 & 2.30278 & 2.30278 & - \\
\hline 1.5 & 2.35074 & 2.35078 & 2.35078 & 2.35708 & 2.83333 & 2.63459 & 2.63476 & 2.63476 & 2.63476 & 2.98075 \\
\hline 2 & 2.73202 & 2.73205 & 2.73205 & 2.73205 & 3 & 2.90843 & 2.99232 & 3 & 3 & 3.5 \\
\hline 2.5 & 3.13704 & 3.13746 & 3.13746 & 3.13746 & 3.3 & 3.38191 & 3.386 & 3.386 & 3.386 & 3.7 \\
\hline 3 & 3.56148 & 3.56155 & 3.56155 & 3.56155 & 3.66667 & 3.79121 & 3.79129 & 3.79129 & 3.79129 & 4 \\
\hline 3.5 & 3.99781 & 3.99999 & 4 & 4 & 4.07143 & 4.21094 & 4.21215 & 4.21221 & 4.21221 & 4.35714 \\
\hline 4 & 4.44941 & 4.44949 & 4.44949 & 4.44949 & 4.5 & 4.64028 & 4.64568 & 4.64575 & 4.64575 & 4.75 \\
\hline 4.5 & 4.90749 & 4.90754 & 4.90754 & 4.90754 & 4.94444 & 4.98792 & 5.08938 & 5.08945 & 5.08945 & 5.16667 \\
\hline 5 & 5.37221 & 5.37228 & 5.37228 & 5.37228 & 5.4 & 5.54026 & 5.54138 & 5.54138 & 5.54138 & 5.6 \\
\hline
\end{tabular}

Table 2: Comparison of the values of $-f^{\prime \prime}(0)$ for different values of $M$ and for injection

\begin{tabular}{|c|c|c|c|c|c|c|c|c|}
\hline \multirow{3}{*}{ K } & \multicolumn{4}{|c|}{$M=1$} & \multicolumn{4}{|c|}{$M=2$} \\
\hline & \multicolumn{2}{|c|}{ Present } & \multirow{2}{*}{$\begin{array}{c}\text { Chakrab } \\
\text { arti and } \\
\text { Gupta } \\
{[3]}\end{array}$} & \multirow[b]{2}{*}{$\begin{array}{l}\text { Pop and } \\
\mathrm{Na}[12]\end{array}$} & \multicolumn{2}{|c|}{ Present } & \multirow{2}{*}{$\begin{array}{c}\text { Chakrabarti } \\
\text { and Gupta } \\
\text { [3] }\end{array}$} & \multirow[b]{2}{*}{$\begin{array}{l}\text { Pop and } \\
\text { Na [12] }\end{array}$} \\
\hline & $10^{\text {th }}$ order & $20^{\text {th }}$ order & & & $10^{\text {th }}$ order & $20^{\text {th }}$ order & & \\
\hline-0.5 & 1.18604 & 1.18614 & 1.18614 & - & 1.49865 & 1.5 & 1.5 & - \\
\hline-1 & 0.99855 & 1 & 1 & - & 1.30271 & 1.30278 & 1.30278 & - \\
\hline-1.5 & 0.85077 & 0.85078 & 0.85078 & 1.20165 & 1.13741 & 1.13746 & 1.13746 & 1.68889 \\
\hline-2 & 0.73198 & 0.73205 & 0.73205 & 0.75 & 0.98793 & 1 & 1 & 1.21875 \\
\hline-2.5 & 0.63741 & 0.63746 & 0.63746 & 0.62592 & 0.8849 & 0.886 & 0.886 & 0.90048 \\
\hline-3 & 0.56151 & 0.56155 & 0.56155 & 0.55144 & 0.79118 & 0.79129 & 0.79129 & 0.77778 \\
\hline-3.5 & 0.49897 & 0.5 & 0.5 & 0.49337 & 0.71189 & 0.71222 & 0.71221 & 0.69863 \\
\hline-4 & 0.44941 & 0.44949 & 0.44949 & 0.44531 & 0.64568 & 0.64575 & 0.64575 & 0.63574 \\
\hline-4.5 & 0.40749 & 0.40753 & 0.40753 & 0.40488 & 0.58930 & 0.58945 & 0.58945 & 0.58253 \\
\hline-5 & 0.37222 & 0.37228 & 0.37228 & 0.37056 & 0.54129 & 0.54138 & 0.54138 & 0.53664 \\
\hline
\end{tabular}


From the Table 1 , it is obvious that the skin friction i.e. $-f^{\prime \prime}(0)$ increases along with increasing values of magnetic parameter $(M)$ and the mass suction parameter $(K>0)$. From the Table 2, it is obvious that the skin friction i.e. $-f^{\prime \prime}(0)$ decreases with increasing values of mass injection parameter $(K<0)$ but decreases with decreasing values of magnetic parameter $(M)$.

Also, from the Tables 1-2, it is obvious that all the values of skin friction derived in the present paper are in good agreement with those obtained by Chakrabarti and Gupta [3] and also with the values of skin friction obtained by Pop and $\mathrm{Na}[12]$ for large suction $(K \gg 1)$ and for large injection $(|K| \gg 1)$ parameters.

From the Fig. 3, it is evident that the velocity profiles decrease alongwith the increasing values of $\eta$. This decrease in velocity profile is more pronounced for increasing values of magnetic parameter. In this case, the mass transfer parameter $K$ has been taken as zero.

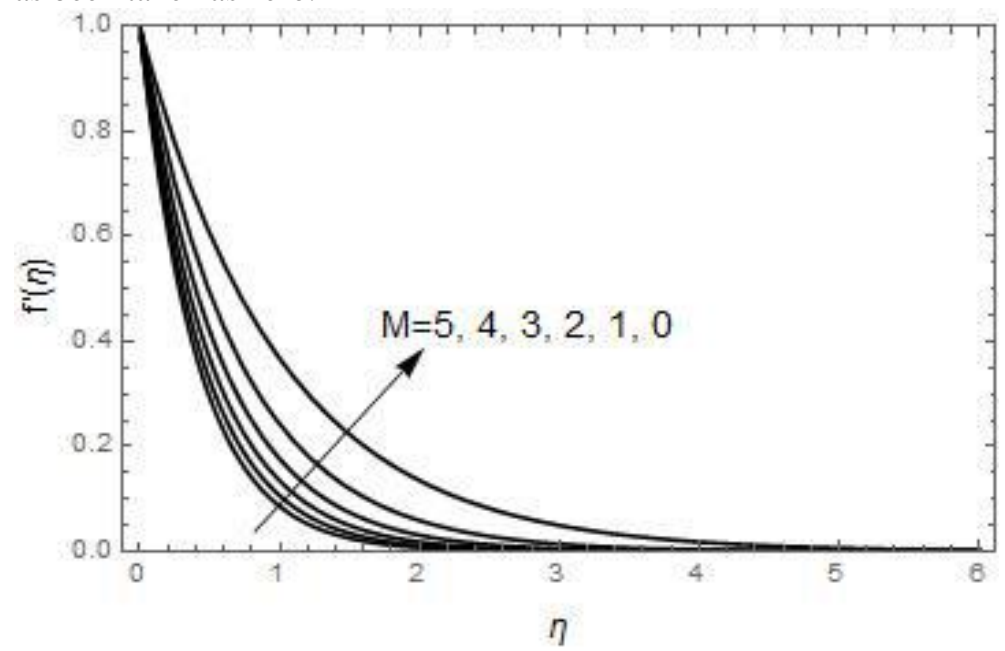

Fig. 3: Velocity profiles in the absence of suction parameter i.e. for $K=0$ for different values of magnetic parameter

In the Fig. 4, the effect of increasing values of magnetic parameter $M$ on the velocity profiles has again been studied. But, unlike the Fig. 3, the value of suction parameter $K$ has been taken as $K=1.5$. Here also, the same trend of decrease in velocity profiles with increasing values of magnetic parameter $M$ has been observed. But, in this case in which the suction parameter (i.e. $K>0$ ) has also been effected, the decrease in velocity profiles is much quicker. As the magnetic parameter $M$ increases, the Lorentz force which opposes the flow also increases, which leads to an enhanced deceleration of the flow.

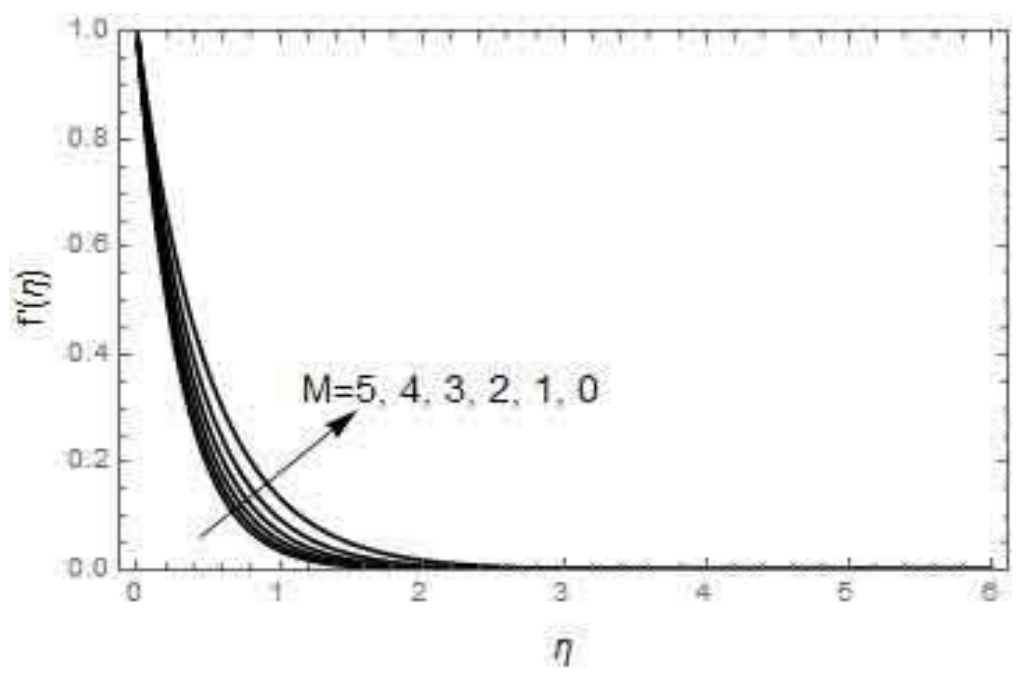

Fig. 4: Velocity profiles for the suction parameter $K=1.5$ and for different values of magnetic parameter

From Fig. 5, it is obvious that like Fig. 4, there occurs a decrease in the velocity profiles with increasing values of magnetic parameter $M$ for a fixed value of injection parameter $K=-1.5$. But, this decrease in the velocity profiles in case of Fig. 5 is slower as compared to that in Fig. 4. 


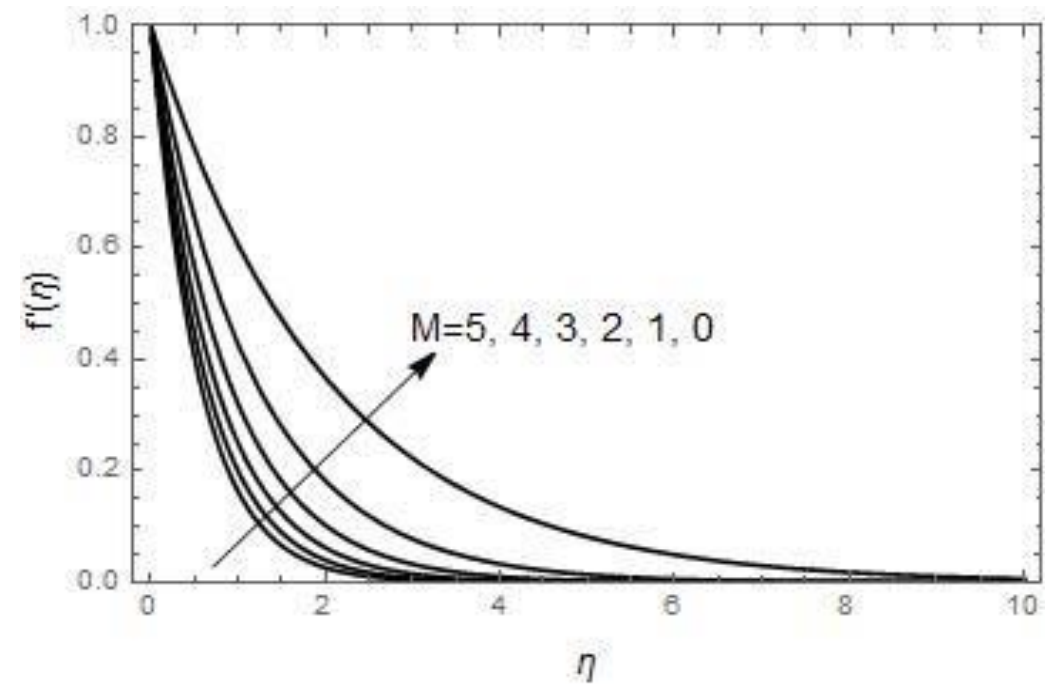

Fig. 5: Velocity profiles for the injection parameter $K=-1.5$ and for different values of magnetic parameter

From Fig. 6, it is evident that the velocity profiles decrease with the increasing values of suction parameter (i.e. $K>0$ ) for $M=0$.

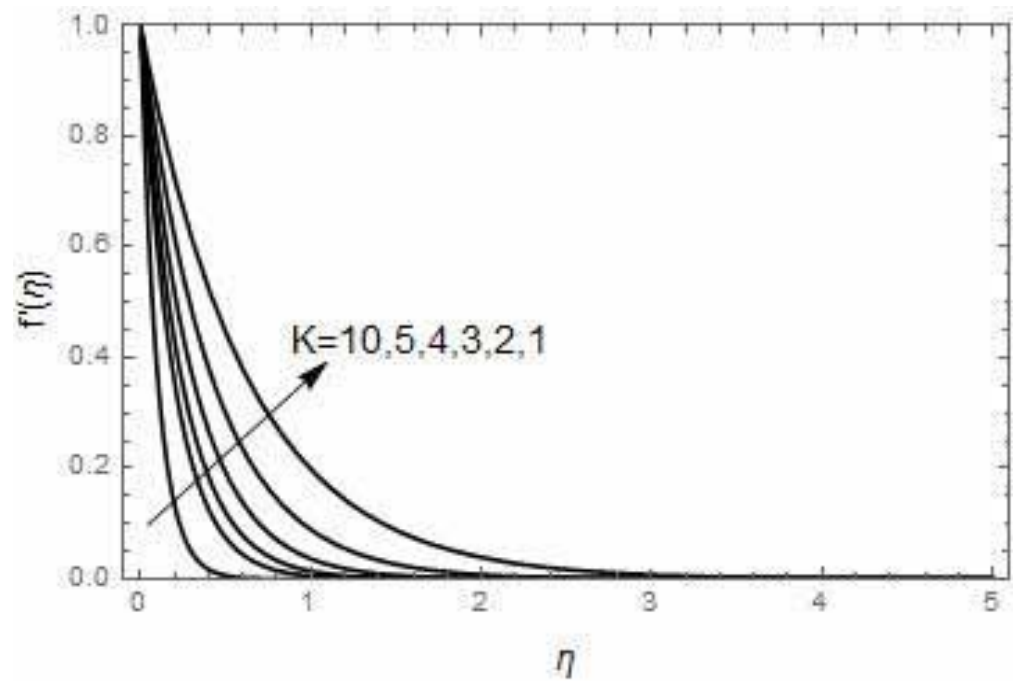

Fig. 6: Velocity profiles in the absence of magnetic parameter i.e. for $M=0$ for different values of suction parameter $K$ $(K>0)$

From Fig. 7, it is clear that there occurs an increase in the velocity profiles with increasing values of injection parameter (i.e. $K<0$ ).

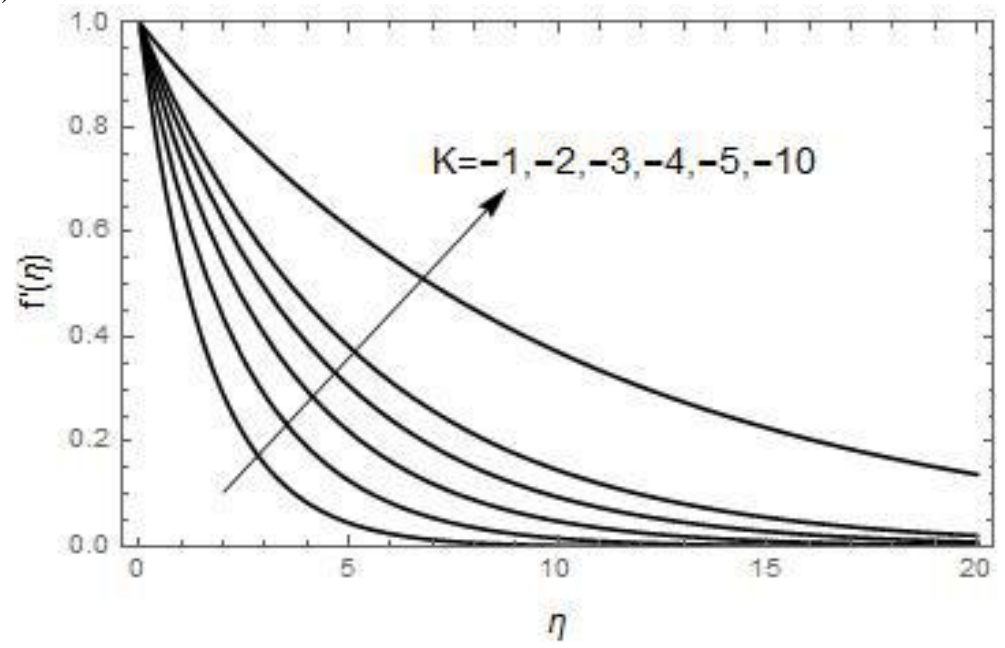

Fig. 7: Velocity profiles in the absence of magnetic parameter i.e. for $M=0$ for different values of injection parameter $K$ $(K<0)$ 


\section{Concluding remarks}

In the present paper, we have discussed the MHD flow of a viscous and incompressible fluid over a stretching permeable surface. An analytical solution in the form of series has successfully been obtained with the use of HAM. The results obtained in the present case have been compared with the available results and have been found compatible with each other. From the tables, it is evident that the skin friction increases with suction and the effects are opposite in case of injection. However, the skin friction increases with $M$ in both the cases of suction and injection.

The perturbation technique used by Pop and $\mathrm{Na}[12]$ is based on the existence of the large suction $(K \gg 1)$ or large injection $(|K| \gg 1)$ parameters. Thus, the perturbation technique used by [12] is not applicable for general suction/injection parameter $K$ especially for small suction/injection parameters. However, the HAM used in the present problem is applicable for general $K$.

The velocity profiles also show a decreasing trend with the increasing values of magnetic parameter for a fixed suction/injection parameter. But, this trend of decrease in case of injection is slower as compared to that in presence of suction, thereby proving that the chances of boundary layer flow becoming more stable in case of injection is less as compared to that in the presence of suction.

\section{Acknowledgement}

One of the authors, Chandrakant N. Guled, would like to express his sense of gratitude to Dr. Babasaheb Ambedkar Technological University, Lonere for providing him with TEQIP-II fellowship for pursuing his Ph.D. programme.

\section{References}

[1] Andersson, H.I., Hansen, O.R., Holmedal, B.: Diffusion of a chemically reactive species from a stretching sheet. International Journal of Heat and Mass Transfer. 37(4), 659-664 (1994)

[2] Pavlov, K.B.: Magnetohydrodynamic flow of an incompressible viscous fluid caused by deformation of a plane surface. Magnitnaya Gidrodinamika. 4, 146-147 (1974)

[3] Chakrabarti, A., Gupta, A.S.: Hydromagnetic flow and heat transfer over a stretching sheet. Quarterly of Applied Mathematics. 37(1), 73-78 (1979)

[4] Vajravelu, K.: Hydromagnetic flow and heat transfer over a continuous, moving, porous, flat surface. Acta Mechanica. 64(3-4), 179-185 (1986)

[5] Takhar, H.S., Raptis, A.A., Perdikis, C.P.: MHD asymmetric flow past a semi-infinite moving plate. Acta Mechanica. 65(1), 287-290 (1987)

[6] Kumari, M., Takhar, H.S., Nath, G.: MHD flow and heat transfer over a stretching surface with prescribed wall temperature or heat flux. Wärme-und Stoffübertragung. 25(6), 331-336 (1990)

[7] Vajravelu, K., Rollins, D.: Heat transfer in an electrically conducting fluid over a stretching surface. International Journal of Non-linear mechanics. 27(2), 265-277 (1992)

[8] Andersson, V.P.H.: MHD flow of a viscoelastic fluid past a stretching surface. Acta Mechanica. 95(14), 227-230 (1992)

[9] Andersson, H.I.: An exact solution of the Navier-Stokes equations for magnetohydrodynamic flow. Acta Mechanica. 113(1-4), 241-244 (1995)

[10] Watanabe, T., Pop, I.: Hall effects on magnetohydrodynamic boundary layer flow over a continuous moving flat plate. Acta Mechanica. 108(1-4), 35-47 (1995)

[11] Lawrence, P.S., Rao, B.N.: Magnetohydrodynamic flow past a semi-infinite moving plate. Acta mechanica. 117(1-4), 159-164 (1996)

[12] Pop, I., Na, T. Y.: A note on MHD flow over a stretching permeable surface. Mechanics Research Communications. 25(3), 263-269 (1998)

[13] Liao, S.J., Pop, I.: Explicit analytic solution for similarity boundary layer equations. International Journal of Heat and Mass Transfer. 47(1), 75-85 (2004)

[14] Hayat, T., Sajid, M.: Analytic solution for axisymmetric flow and heat transfer of a second grade fluid past a stretching sheet. International Journal of Heat and Mass Transfer. 50(1), 75-84 (2007) 
[15] Hayat, T., Abbas, Z., Sajid, M., Asghar, S.: The influence of thermal radiation on MHD flow of a second grade fluid. International Journal of Heat and Mass Transfer. 50(5), 931-941 (2007)

[16] Hayat, T., Sajid, M.: Homotopy analysis of MHD boundary layer flow of an upper-convected Maxwell fluid. International Journal of Engineering Science. 45(2), 393-401 (2007)

[17] Hayat, T., Qasim, M., Abbas, Z.: Homotopy solution for the unsteady three-dimensional MHD flow and mass transfer in a porous space. Communications in Nonlinear Science and Numerical Simulation. 15(9), 2375-2387 (2010)

[18] Liao, S.J.: An approximate solution technique not depending on small parameters: a special example. International Journal of Non-Linear Mechanics, 30(3), 371-380 (1995)

[19] Cheng, J., Liao, S.J., Pop, I.: Analytic series solution for unsteady mixed convection boundary layer flow near the stagnation point on a vertical surface in a porous medium. Transport in porous media. 61(3), 365-379 (2005)

[20] Wang, C., Pop, I.: Analysis of the flow of a power-law fluid film on an unsteady stretching surface by means of homotopy analysis method. Journal of Non-Newtonian Fluid Mechanics. 138(2), 161-172 (2006)

[21] Xu, H., Liao, S.J., Pop, I.: Series solutions of unsteady three-dimensional MHD flow and heat transfer in the boundary layer over an impulsively stretching plate. European Journal of Mechanics-B/Fluids. 26(1), 15-27 (2007)

[22] Cheng, J., Liao, S.J., Mohapatra, R. N., Vajravelu, K.: Series solutions of nano boundary layer flows by means of the homotopy analysis method. Journal of Mathematical Analysis and Applications. 343(1), 233-245 (2008)

[23] Xu, H., Liao, S.J., Pop, I.: Series solutions of unsteady free convection flow in the stagnation-point region of a three-dimensional body. International Journal of Thermal Sciences. 47(5), 600-608 (2008)

[24] Fan, T., Xu, H., Pop, I.: Unsteady stagnation flow and heat transfer towards a shrinking sheet. International Communications in Heat and Mass Transfer. 37(10), 1440-1446 (2010)

[25] Wang, Z.K., Kao, T.A.: An introduction to homotopy methods. Chongqing: Chongqing Publishing House (1991)

[26] Liao, S.J.: The proposed homotopy analysis technique for the solution of nonlinear problems. Ph. D. Thesis. Shanghai Jiao Tong University (1992)

[27] Liao, S.J.: Notes on the homotopy analysis method: some definitions and theorems. Communications in Nonlinear Science and Numerical Simulation. 14(4), 983-997 (2009)

[28] Shercliff, J.A.: Textbook of magnetohydrodynamics (1965)

[29] Liao, S.J.: Beyond perturbation: introduction to the homotopy analysis method. Chapman I\& Hall/ CRC press, Boca Raton (2003)

[30] Saraf, A.R., Sadaiah, M. and Devkare, S.: Optimization of photochemical machining. Int J of Engg Sci and Tech., 1(3), 7108-7116 (2011).

[31] Saraf, A.R., Misal, N.D. and Sadaiah, M.: Mathematical modelling and optimization of photochemical machining. Adv Mat Res, 548, 617-622 (2012)

[32] Saraf, A.R. and Sadaiah, M.: Application of artificial intelligence for the prediction of undercut in photochemical machining. Int J of Mech and Mfg Syst, 6(2), 183-194 (2013).

[33] Saraf, A.R. and Sadaiah, M.: Photochemical Machining of SS304, Int. J of Adv in Sci and Tech, 3(6), 71-85 (2011).

[34] Saraf, A.R. and M.Sadaiah:. Photochemical Machining of SS316L, Mfg Tech Today, 11(7), 5-9 (2012)

[35] Saraf, A.R. and Sadaiah, M. :Magnetic field assisted photochemical machining of SS316L" accepted in the journal "Materials and Manufacturing Processes" Taylor and Francis. ( ID: 1198014 DOI:10.1080/10426914.2016.1198014)

[36] Saraf, A.R. and Sadaiah, M. : Photochemical Machining of a Cardiovascular Stent" accepted in the journal "Materials and Manufacturing Processes" Taylor and Francis. (ID: 1198025 DOI:10.1080/10426914.2016.1198025)

[37] Guled, C.N. and Singh, B.B., 2016. Homotopy Analysis of the Radiation Effect on MHD Flow with Heat and Mass Transfer due to a Point Sink. Adv in Math Phy, 2016, 1-14 (2016). 